\title{
Analyzing and evaluating the energy efficiency based on multi-5G small cells with a mm-waves in the next generation cellular networks
}

\author{
Mohammed H. Alsharif ${ }^{1}$, Khalid Yahya ${ }^{2}$, Shehzad Ashraf Chaudhry ${ }^{3}$ \\ ${ }^{1,2}$ Department of Electrical and Electronics Engineering, Faculty of Engineering and Architecture, \\ Istanbul Gelisim University, Turkey \\ ${ }^{3}$ Department of Computer Engineering, Faculty of Engineering and Architecture, Istanbul Gelisim University, Turkey
}

\begin{tabular}{l} 
Article Info \\
\hline Article history: \\
Received Sep 30, 2019 \\
Revised Jan 14, 2020 \\
Accepted Feb 1, 2020 \\
\hline
\end{tabular}

\section{Keywords:}

Data rate

Energy efficiency

Fifth generation $(5 \mathrm{G})$

MCS

Small cell

\begin{abstract}
This paper evaluates the impact of multi-5G small cell systems on the energy efficiency (EE) in a Fifth Generation (5G) of cellular networks. Both the proposed model and the analysis of the EE in this study take into account (i) the path losses, fading, and shadowing that affect the received signal at the user equipment (UE) within the same cell, and (ii) the interference effects of adjacent cells. In addition, the concepts of new technologies such as large MIMO in millimeter range communication have also been considered. The simulation results show that the interference from adjacent cells can degrade the EE of a multi-cell cellular network. With the high interference the number of bits that will be transferred per joule of energy is $1.29 \mathrm{Mb} / \mathrm{J}$ with a $0.25 \mathrm{GHz}$ bandwidth and 16 transmit antennas. While, with a $1 \mathrm{GHz}$ bandwidth the transfer rate increases to $5.17 \mathrm{Mb} / \mathrm{J}$. Whereas, with 64 transmit antennas the EE improved to $5.17 \mathrm{Mb} / \mathrm{J}$ with a $0.25 \mathrm{GHz} \mathrm{BW}$ and $20.70 \mathrm{Mb} / \mathrm{J}$ with a $1 \mathrm{GHz} \mathrm{BW}$. These results provide insight into the impact of the number of antennas in millimeter range communication and the interference from adjacent cells on achieving real gains in the EE of multi-5G small cells cellular network.
\end{abstract}

Copyright $@ 2020$ Institute of Advanced Engineering and Science. All rights reserved.

\section{Corresponding Author:}

Mohammed H. Alsharif,

Department of Electrical and Electronics Engineering,

Istanbul Gelisim University,

İstanbul, Avc1lar 34310, Turkey.

Email: malsharif@gelisim.edu.tr

\section{INTRODUCTION}

The mobile subscriptions grew around 4\% between Q1 2016 and Q1 2017, to reach 7.6 billion in Q1 2017; while mobile data traffic grew around 70\% in the same period, to reach around 9.7 ExaBytes (EB) per month in Q1 2017; the reason behind this increase is a continuous development in cellular networks and its ability to meet mobile subscriber needs and provide high data speed rates. Accordingly, it is expected that the mobile subscriptions are growing to be 9 billion by 2022, with mobile data traffic approximately up to 71 $\mathrm{EB} /$ month, where that $75 \%$ will be for mobile video traffic [1]. These challenges have been a crucial step towards the fifth generation of cellular networks, which it is expected to be deployed by 2020 [2].

$5 \mathrm{G}$ will have to fulfil many requirements, and a critical one is to deliver high network energy performance [3]. High energy performance requires a fundamental change of design principles and implementation practices within the mobile telecom industry. Thus, 5G technology adopts a set of new technologies; a detailed survey on $5 \mathrm{G}$ networks has been presented in [4]. The intensive deployment of 5G small cells with mm-waves along with large MIMO will play a significant role in developing 5G cellular network, to overcome the explosive increase in mobile traffic and improve EE of the network, as well as 
throughput and a prolong battery life of mobile phone [5]. In 2015, more than 40,000 small cells were deployed [6], and this number is expected to increase in the future upon the deployment of 5G technology [7]. As shown in [8], the number of 5G small cells is anticipated to become four times larger than the number of existing macro sites to cover the same service area. The number of bits transmitted per joule of energy, called energy EE, is used as a performance measure in a green cellular communication system. EE metrics have been discussed in [9, 10]. Since that the performance (the number of bits transmitted) of cellular communication system has been mainly limited by the interference from adjacent cells, as well as by the path losses, fading, and shadowing. Hence, evaluate the impact of multi-cells system on the EE in cellular networks is considered an important issue and need to more investigation, which represents a real cellular network case. Therefore, the key contribution of this paper is a modelling and analyzing EE based on multi-5G small cells of cellular network system to promote the concept of green communication in the next generation network. The concepts of new technologies such as large MIMO (16, 32, and 64 antennas) in millimeter range communication $(28 \mathrm{GHz})$ has been incorporated in the proposed model.

The rest of this paper is organized as follows. Section 2 includes related works. The system model is described in Section 3. The mathematical formulations that considered in this study are given in Section 4. The simulation setup is provided in Section 5. Results and discussion in Section 6. Finally, conclusions are drawn in Section 7.

\section{RELATED WORKS}

The fundamental trade-off between energy efficiency in terms of the total number of bits transmitted per Joule per terminal receiving service of energy spent, and spectral efficiency, has been examined in [11, 12]. Authors in [11] have conducted the trade-off for three cases: (i) A reference system with one single antenna serving a single UE; (ii) A system with 100 antennas serving a single UE using conventional beamforming; (iii) A large MIMO simultaneously serving UE. The simulation results shown that a large MIMO system with 100 antennas an efficient in term of energy efficiency. In addition, the SE- EE trade-off is developed in [13] based on the asymptotic SE and the realistic power consumption model for large MIMO system. In the same context, the simulations that have been conducted in [11] shown that each single-antenna user in a large MIMO system can scale down its transmit power proportional to the number of antennas at the BS with perfect channel state information (CSI) or to the square root of the number of BS antennas with imperfect CSI, to get the same performance as a corresponding single-input single-output (SISO) system. Moreover, the relationship between the energy efficiency and spectrum efficiency in a two-cell LTE cellular network, and the impact of multi-antenna on the energy efficiency of cellular network, investigated in $[14,15]$. However, these studies were address on the EE issue into the legacy cellular networks; and did not address the issue of EE in the next cellular generation (5G), which it is expected to be deployed by 2020 . To continue the vision and promote the concept of green communication and a step towards achieving a green and sustainable 5G cellular networks. This study examined EE issue on multi-5G small cells of cellular network system in millimeter range communication; with consider the interference from adjacent cells, as well as by the path losses, fading, and shadowing.

\section{SYSTEM MODEL}

As shown in Figure 1, the BS coverage range is configured as a typical hexagonal cell. The main inter-interference is assumed to be transmitted from two adjacent cells. The system model is used to study the impact of multi-5G small cell systems on the EE of cellular network. The EE, measured in bits per joule, is defined as the total amount data delivered (in bits per second) divided by the total power consumed by the BS (in watts). The main phenomena that affect on the data delivered (bits per second) from BS1 to UE are: (i) the path losses, fading, and shadowing within the same cell (PL_mod_SUI), and (ii) the interinterference effects of adjacent cells BS2 and BS3 (PI2 and PI3). While, the total power consumed by the BS1 is a directly proportional to the transmission power of BS1 (Ptx_BS1); and the Ptx_BS1 depends on the radius of coverage and the signal propagation fading. More details about the mathematical models will be given in the following section.

\section{MATHEMATICAL MODEL}

\subsection{Energy efficiency model}

Based on the $5 \mathrm{G}$ cellular network system model shown in Figure 1, the EE mathematical model as stated below, 


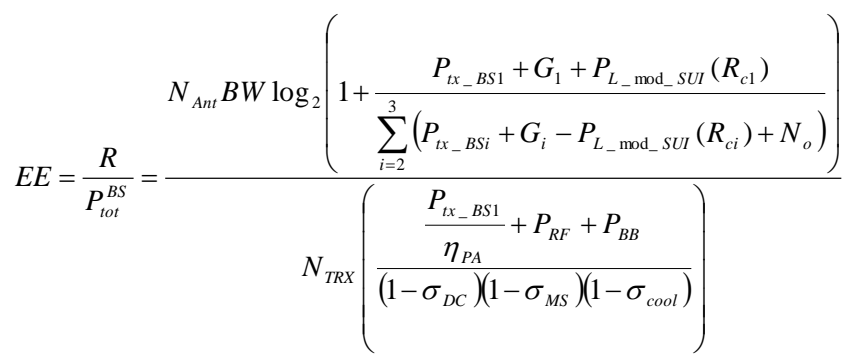

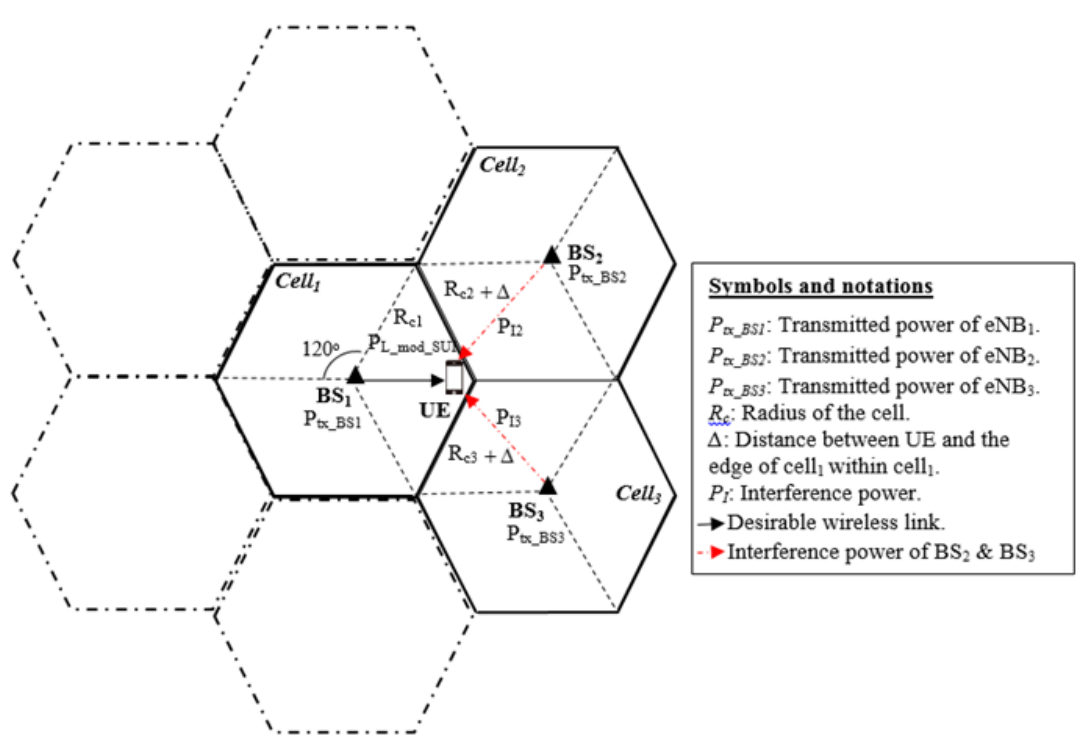

Figure 1. System model of multi-5G small cells

In the EE mathematical model given in (1), the path loss, fading, shadowing, and the interference effects from adjacent cells are considered. However, for ease of understanding, this EE model is simplified and explained in the following paragraphs. In a communication system, the EE, measured in bits per joule, and expressed mathematically [10],

$$
E E=\frac{R}{P_{t o t}^{B S}}
$$

where $R$ is the total data delivered to the UE, and $P_{t o t}^{B S}$ is the total power consumed. $P_{t o t}^{B S}$ can be calculated by the following formula [16],

$$
P_{t o t}^{B S}=N_{T R X}\left(\frac{\frac{P_{t x_{-} B S 1}}{\eta_{P A}}+P_{R F}+P_{B B}}{\left(1-\sigma_{D C}\right)\left(1-\sigma_{M S}\right)\left(1-\sigma_{C o o l}\right)}\right)
$$

where $\sigma_{D C}$ is the DC-DC regulator losses, $\sigma_{M S}$ is the main power supply losses, and $\sigma c o o l$ is the air-conditioner losses. However, by introducing RRHs, feeder losses are avoided by mounting the power amplifier (PA) close to the transmit antenna and using an optical fiber cable. In addition, cooling (air-conditioner) losses are omitted in small BS [16]. $P_{t x_{-} B S 1}$ is the output power per transmit antenna, $P_{R F}$ is the radio frequency power, and $P_{B B}$ is the baseband power. Since that the C-RAN architecture is considered in this study, PBB is omitted, because a baseband unit is installed in the central office. $\eta_{P A}$ is the PA power efficiency, and $N_{T R X}$ is the total transceiver antennas in the site. 


\subsection{Data rate}

As known of Shannon theory, the data rate is directly proportional with the antennas and bandwidth, and logarithmically proportional with SINR [10],

$$
R=N_{\text {Ant }} B W \log _{2}(1+\operatorname{SINR})
$$

where $B W$ is the bandwidth; and SINR is the signal to interference plus noise ratio, and can be calculated by the following formula,

$$
\operatorname{SINR}=\frac{P_{r x}}{\sum_{i=2}^{3} P_{I_{i}}+N_{o}}
$$

where $P_{I}$ is the interference power of the neighbour $\mathrm{BS}_{2}$ and $\mathrm{BS}_{3}$ (which are denoted as $P_{I 2}$ and $P_{I 3}$, based on Figure $1, N_{o}$ is the noise in wireless channels; and $P_{r x}$ is the received power at UE

\subsection{G propagation model}

The basic propagation model of the received power $\left(\mathrm{P}_{\mathrm{rx}}\right)$ as stated below [17],

$$
P_{r x}=P_{t x_{-} B S I}+G_{1}-P_{L_{m o d} d_{S I}}\left(R_{c 1}\right)
$$

where G1 is the antenna gain at BS1, and PL_mod_SUI is the path loss model for 5G small cell.

The transmission power of BS1 $\left(P_{t x_{B} B S I}\right)$, depends on two main factors: (ii) the required coverage area, and (ii) the surrounding environment (propagation fading). To simplify the model derivation, the macro BS transmission power is normalized as $P_{o}=40 \mathrm{~W}$ with the coverage radius $R_{o}=1 \mathrm{~km}$. Similarly, the $P_{t x_{-} B S I}$ with coverage radius $\mathrm{Rc} 1$ is denoted by [18],

$$
P_{t x_{-} B S I}=P_{o} x\left(\frac{R_{C 1}}{R_{o}}\right)^{\alpha}
$$

The mm-wave spectrum is very sensitive to losses due to reflection and diffraction, which greatly depend on the material and the surface. Moreover, reflection and diffraction reduce the range of mm-waves and decrease the ability of a mm-wave signal to penetrate buildings (e.g., brick and concrete) and most solid materials (the attenuations for different materials are given in $[19,20]$. In this study, the modified Stanford University Interim (SUI) path loss model $\left(P_{L_{-} \text {mod_SUI }}\right)$ for a frequency of $28 \mathrm{GHz}$ for Non-Line of Sight (NLOS) is proposed, as given in (8). This mathematical model (the modified SUI path loss model) is constructed based on extensive empirical measurements [8],

$$
P_{L_{\text {mod }} S U I}\left(R_{c 1}\right)=\alpha_{N L O S} x\left(P_{L_{S U I}}\left(R_{c 1}\right)-P_{L_{S U I}}(d o)\right)+P_{L}(d o)+X_{o}
$$

where $\alpha_{N L O S}$ is the mean slope correction factor obtained directly from the NLOS empirical results, $P_{L_{S U I}}\left(R_{C 1}\right)$ is an original SUI model at a distance $R_{c 1}, P_{L_{S U I}}(d o)$ is an original SUI model at a reference distance $d o, P_{L}(d o)$ is the free space path loss in $\mathrm{dB}$ at $d o$, and $X \sigma$ is a typical lognormal random shadowing variable with $0 \mathrm{~dB}$ mean and standard deviation $\sigma$. The original SUI model $P_{L_{-} S U I(R c l)}$ for a frequency above 2 $\mathrm{GHz}$ is [8],

$$
P_{L S U I}\left(R_{c 1}\right)=P_{L}\left(d_{o}\right)+10 n \log _{10}\left(\frac{R_{c 1}}{d_{o}}\right)+X_{f c}+X_{R X}+X_{\sigma}
$$

where,

$$
\begin{aligned}
& P_{L}\left(d_{o}\right)=20 \log _{10}\left(\frac{4 \pi d_{o}}{\lambda}\right) \\
& n=a-b \cdot h_{T X_{-} B S 1}+\frac{C}{h_{T X_{-} B S 1}}
\end{aligned}
$$




$$
\begin{aligned}
& X_{f c}=6 \log _{10}\left(\frac{f_{M H z}}{2000}\right) \\
& X_{R X}=-10.8 \log _{10}\left(\frac{h_{R X}}{2}\right)
\end{aligned}
$$

where $\lambda$, Xfc, and XRX denote the carrier wavelength, the correction factors for frequency, and receiver heights, respectively. $f_{M H z}$ is the carrier frequency. $h_{T X}$ and $h_{R X}$ is the transmitter and receiver antenna heights, respectively. The parameters $\mathrm{a}, \mathrm{b}$, and $\mathrm{c}$ are constants used to model the terrain types encountered in the service area. The SUI terrain type A is considered, with parameters given as $a=4.6, b=0.0075$, and $\mathrm{c}=12.6[8]$.

The interference power of the adjacent cells that affect the received signal at the UE can be expressed mathematically as,

$$
P_{I}=\sum_{i=2}^{3}\left(P_{t x-B S i}+G_{i}-P_{L_{\text {mod }} S U}\left(R_{c i}\right)\right)
$$

The transmission power of the BS2 and BS3, and the modified-SUI NLOS path loss model are expressed similarly to BS1, as given in the following equations,

$$
\begin{aligned}
& P_{t x_{-} B S i}=\sum_{i=2}^{3} P_{o} x\left(R_{c i} / R_{o}\right)^{\alpha} \\
& P_{L_{\text {mod }} \text { SUI }}\left(R_{c i}\right)=\alpha_{N L O S} x\left(P_{L_{S U I}}\left(R_{c i}+\Delta\right)-P_{L_{S U I}}\left(d_{o}\right)\right)+P_{L}\left(d_{o}\right)+X_{\sigma}
\end{aligned}
$$

where $\Delta$ represents the distance between the UE and the edge of the cell1 within cell1. For example, if the UE is being determined at the edge of cell1, $\Delta$ will equal zero

\section{SIMULATION SETUP}

\begin{tabular}{|c|c|c|c|}
\hline Item & Parameter & Acronym & $\mathrm{Cell}_{1}$ \\
\hline \multirow[t]{4}{*}{ Network } & Carrier frequency & $f_{c}$ & $28 \mathrm{GHz}$ \\
\hline & Bandwidth & $B W$ & $0.25,0.5,1 \mathrm{GHz}$ \\
\hline & Max. cell radius & $R_{c l}$ & $200 \mathrm{~m}$ \\
\hline & Reference distance & $d_{o}$ & $1 \mathrm{~m}$ \\
\hline \multirow[t]{12}{*}{ BS } & eNB transmission power & $P_{t x_{-} B S I}$ & $\begin{array}{l}1.6 \mathrm{~W} \\
32.04 \mathrm{dBm}\end{array}$ \\
\hline & (PA) power efficiency & $\eta_{P A}$ & $38.8 \%$ \\
\hline & Radio frequency & $P_{R F}^{D C}$ & $0.7 \mathrm{~W}$ \\
\hline & Baseband & $P_{B B}^{D C}$ & C-RAN \\
\hline & DC-DC power loss & $\sigma_{D C}$ & $8 \%$ \\
\hline & Main supply loss & $\sigma_{M S}$ & $10 \%$ \\
\hline & Cooling & $\sigma_{\text {Cool }}$ & No needed (small cell) \\
\hline & eNB antenna height & $h_{T X_{-} B S I}$ & $7 \mathrm{~m}$ \\
\hline & Tx antenna gain & $G_{I}$ & $7 \mathrm{~dB}$ \\
\hline & Number of antennas & $N_{\text {Ant }}$ & $16,32,64$ \\
\hline & Noise figure & $N_{f}$ & $9 \mathrm{~dB}$ \\
\hline & UE antenna height & $h_{U E}$ & $1.5 \mathrm{~m}$ \\
\hline Propagation & Morphology & & \\
\hline \multirow[t]{5}{*}{ losses } & Propagation model & $P_{L_{-} \text {mod_SUI }}$ & Modified SUI-NLOS \\
\hline & Slope correction factor & $\alpha_{N L O S}$ & 0.71 \\
\hline & AWGN & $N_{o}$ & $6 \mathrm{~dB}$ \\
\hline & Shadow fading margin & $X_{\sigma}$ & $6 \mathrm{~dB}$ \\
\hline & Exponent path loss & $\alpha$ & 3.2 \\
\hline
\end{tabular}

The simulation layout is given in Figure 1. The modified SUI model at $28 \mathrm{GHz}$ is considered in this study; additional details about the simulation parameters that are used in the present study are given in Table 1 . Note that the simulation parameters for both cell $l_{2}$ and cell $l_{3}$ are similar to cell $_{1}$.

Table 1. List of simulation parameters 


\section{RESULTS AND DISCUSSION}

The received power level is commonly used in wireless communication as a measure of the quality of wireless connections because each radio receiver can only detect and decode signals with strengths greater than the minimum receiver sensitivity power. This section first discusses the effects of the path loss, fading and shadowing on the received power level at the UE within cell $l_{1}$, as well as the interference effects from adjacent cells ( cell $_{2}$ and cell ${ }_{3}$ ) on the received power level at the UE over different cell radii. Because the EE is a function of both the data rate and the total BS power consumption, the second part of the discussion focuses on the data rate that will be delivered to the UEs under the effects of propagation path loss, multi-path (small-scale) fading, shadow (large-scale) fading and the interference power of the adjacent cells. The final part of this section evaluates the EE based on the proposed multi-LTE macro BSs system model. The received signal power decreases rapidly as the transmit-receive distance (radius of cell) increases $\left(P_{r x}(r)=P_{r x} r^{-\alpha}\right.$. This power decrease is because the path loss, fading and shadowing within the cell, as well as the interference of adjacent cells and noise, are increasing. Figure 2 shows the effect of both the path loss, fading and shadowing within cell ${ }_{1}$ and of the interference of adjacent cells $\left(\right.$ cell $_{2}$ and cell $\left.{ }_{3}\right)$ on the received power level at the UE versus cell radii.

It is clear that when the radius increases, the received power level of $\mathrm{BS}_{1}$ decreases and the interference power of $\mathrm{BS}_{2}$ and $\mathrm{BS}_{3}$ increases, reaching a maximum at the edge of cell. This can be expressed as the SINR given in (5) and defined as the ratio between the received power level of $\mathrm{BS}_{1}$ and the interference power of $\mathrm{BS}_{2}$ and $\mathrm{BS}_{3}$ plus noise. Figure 3 shows the SINR over different radii. Where that the $\mathrm{mm}$-wave spectrum $(28 \mathrm{GHz})$ is very sensitive to the losses, SINR at edge of cell1 drops to $-10.6 \mathrm{~dB}$.

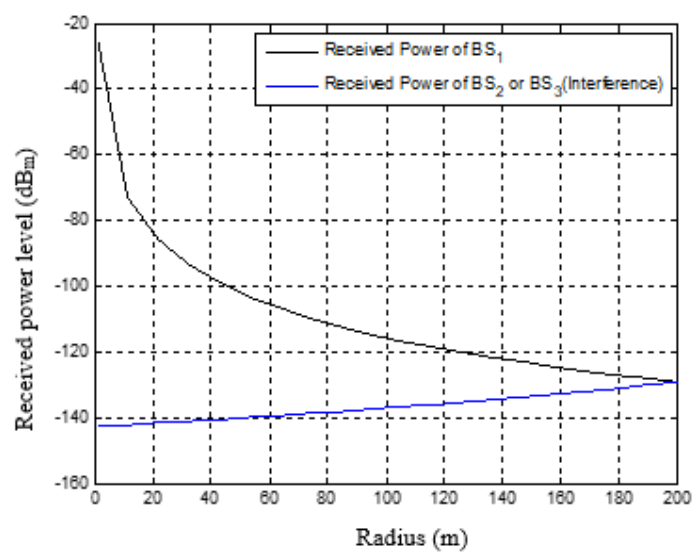

Figure 2. Cell radii versus received power level at $\mathrm{P}_{\mathrm{tx} \_\mathrm{BS} 1}=\mathrm{P}_{\mathrm{tx}_{\_} \mathrm{BS} 2}=\mathrm{P}_{\mathrm{tx}_{\_} \mathrm{BS} 3}=32.04 \mathrm{~dB}_{\mathrm{m}}$

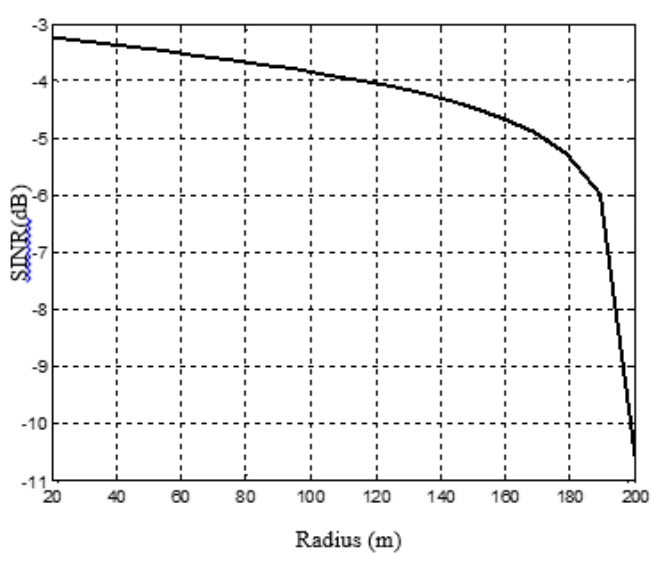

Figure 3. Cell radii versus SINR

As shown in (4), the data rate is directly proportional with the antennas and bandwidth, and logarithmically proportional with SINR. Hence, the key 5G considerations are focused on the physical layer (PHY), intensive deployment of 5G small cells with mm-waves along with large MIMO:

- 5G Small cells, radio coverage of 5G small cell BSs is $200 \mathrm{~m}$. However, a simulation of network parameters for different cell radii is performed. As shown in Figure 3, when the distance between the BS and UEs has increased, SINR is degraded, which considered as the metric for quality of wireless connections. Thus, the worst received wireless signal of $5 \mathrm{G}$ small cell BS is $-10.6 \mathrm{~dB}$ at the edge of the cell1; which will reflect on the data rate as shown in (4) as well as on the amount of data transferred in the joule, as shown in (2); due to using a low order of modulation technique.

- Millimeter wave (mm-wave): a three BW values have investigated to cover the most potential standards, which are $0.25,0.5$, and $1 \mathrm{GHz}$. Based on (4), Figure 4 shows the relationship between the data rate and the SINR values for different bandwidths and for 64 transmit antennas.

The following analysis discusses the data rate at the edge of cell ${ }_{1}$ over different of the BW values. As shown in Figure 5, the data rate up to 1.93, 3.86, and 7.72 Gbps with BW 0.25, 0.5, and $1 \mathrm{GHz}$, respectively. Which obtained based on (4). It is clear that at the largest BW the data rate amounted four times at the lowest BW. These results mean that the 5G small cell can support a new high-speed data application, including multimedia communications, online gaming, and high-quality video streaming. Anyway, since that the EE is a function of the data rate and BS power consumption. The EE performance versus the SINR for different BWs is shown in Figure 5, based on the multi-5G small cells network system proposed in Figure 3.

Analyzing and evaluating the energy efficiency based on multi-5G small cells ... (Mohammed H. Alsharif) 
The total power consumption in this type of system grows proportionally with transmission power of the base station $P_{t x_{-} B S}$ as is shown in (3). As shown in Figure 5, the EE achieved at a $0.25 \mathrm{GHz} \mathrm{BW}$ and 64 antennas at the edge of the cell ${ }_{1}$, where the SINR is $-10.6 \mathrm{~dB}$, can be up to $5.18 \mathrm{Mbits} / \mathrm{J}$. This result computed using (2), a data rate of 1.93 Gbps taken from Figure 4, and by dividing by the total power consumption of $372.86 \mathrm{~W}$ (according to as shown in (3) $64 \times[(4.12+0.7) /(1-0.08)(1-0.1)])$. However, with a $1 \mathrm{GHz} \mathrm{BW}$ and 64 antennas, the EE can be as high as $20.70 \mathrm{Mbits} / \mathrm{J}$, resulting in data rates of up to $7.72 \mathrm{Gbps}$, as shown in Figure 5.

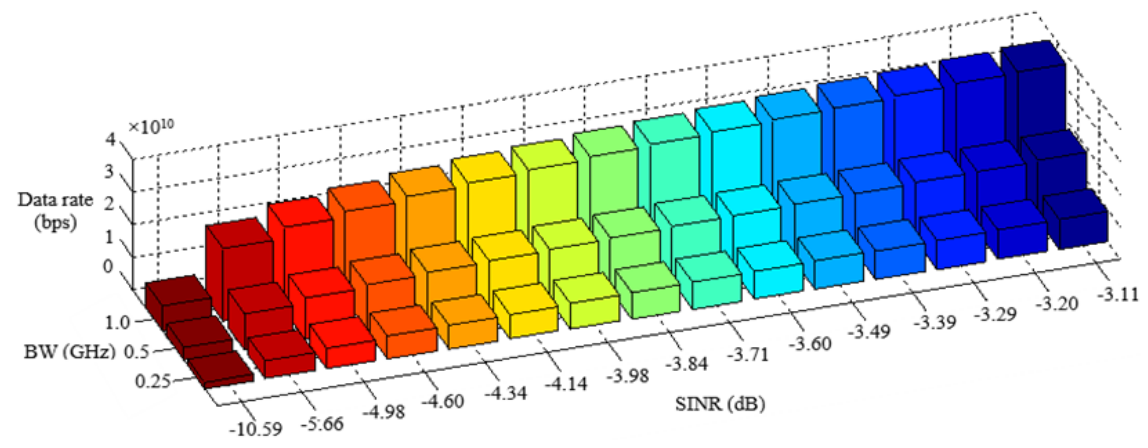

Figure 4. Data rate versus different SINR values for different BWs at $\mathrm{P}_{\mathrm{tx} \_\mathrm{BS} 1}=32.04 \mathrm{~dB}_{\mathrm{m}}$ and $\mathrm{N}_{\text {Ant }}=64$

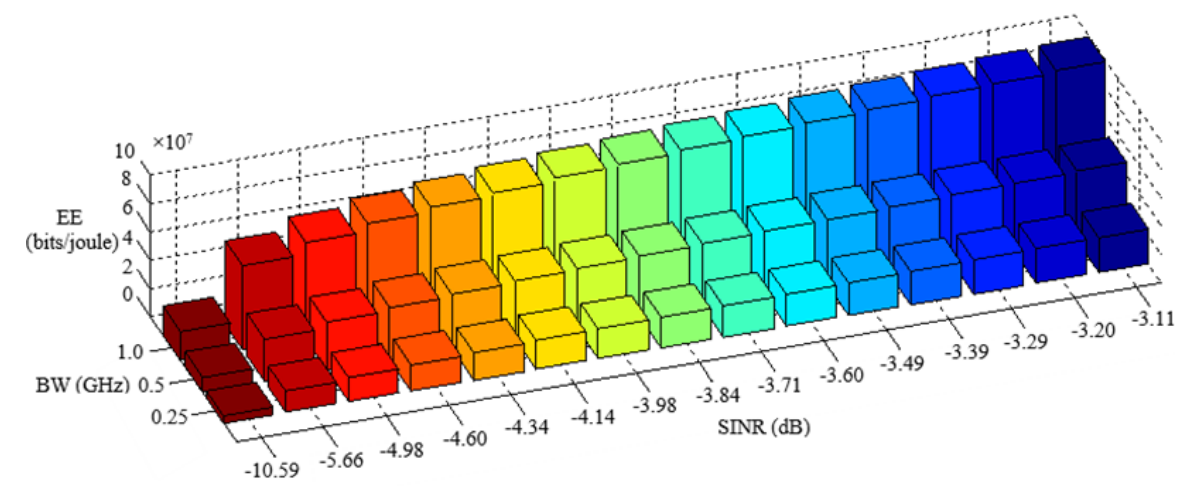

Figure 5. Energy efficiency versus different SINR values for different BWs at $\mathrm{P}_{\mathrm{tx} \_\mathrm{BS} 1}=\mathrm{P}_{\mathrm{tx} \_\mathrm{BS} 2}=\mathrm{P}_{\mathrm{tx} \_\mathrm{BS} 3}=32.04 \mathrm{~dB}_{\mathrm{m}}$ and $\mathrm{N}_{\mathrm{Ant}}=64$

- Large MIMO, is another method that can increase the capacity and EE [21-23]. Using Large MIMO technology, providing high multiplexing as well as array gain at the same time. Large MIMO technology is not only spectrum efficient but energy efficient as well. As the number of BS antennas increases, the received SINR increases linearly [24-26]. Figure 6 summarizes the data rate that can be achieved with various numbers of antennas for different BWs at the edge of cell $1_{1}$.

At the edge of cell $1_{1}$ (radius tual $200 \mathrm{~m}$ ) where the SINR is the lowest $(-10.6 \mathrm{~dB})$, the total data rate with a $0.25 \mathrm{GHz}$ BW and 32 antennas can be up to $0.96 \mathrm{Gbps}$. With a $1 \mathrm{GHz}$ BW the total data rate can be up to $3.86 \mathrm{Gbps}$ with the same number of antennas. However, the multiple signal paths due to multiple antennas at the transmitter are responsible for the large throughput. It is clear that when using 64 antennas, the data rate (1.93 Gbps with $0.25 \mathrm{GHz} \mathrm{BW}$, and $7.72 \mathrm{Gbps}$ with $1 \mathrm{GHz} \mathrm{BW}$, respectively) is 2 times greater compared with using 32 antennas.

The data rate increases with an increasing number of antennas, and the EE is a function of the data rate (as shown by (2)). Therefore, the EE improves with a larger BW size and a larger number of antennas. Figure 7 shows EE versus the number of antennas for different BWs at the edge of cell $1_{1}$. At the edge of cell 1 where the SINR is the lowest $(-10.6 \mathrm{~dB})$, the total EE with a $0.25 \mathrm{GHz}$ BW and 16 antennas can be up to $1.29 \mathrm{Mb} \backslash \mathrm{j}$. With a $1 \mathrm{GHz} \mathrm{BW}$ the total EE can be up to $5.17 \mathrm{Mb} \backslash \mathrm{j}$ with the same number of antennas. While, with 32 antennas the total EE with a $0.25 \mathrm{GHz} \mathrm{BW}$ can be up to $2.59 \mathrm{Mb} \backslash \mathrm{j}$, and with a $1 \mathrm{GHz} \mathrm{BW}$ the can be up to $10.35 \mathrm{Mb} \backslash \mathrm{j}$. Moreover, the EE values increased 4 times with a 64 antennas compare with 16 antennas, which amounted $5.17 \mathrm{Mblj}$ with a $0.25 \mathrm{GHz} \mathrm{BW}$ and $20.70 \mathrm{Mblj}$ with a $1 \mathrm{GHz} \mathrm{BW}$. 


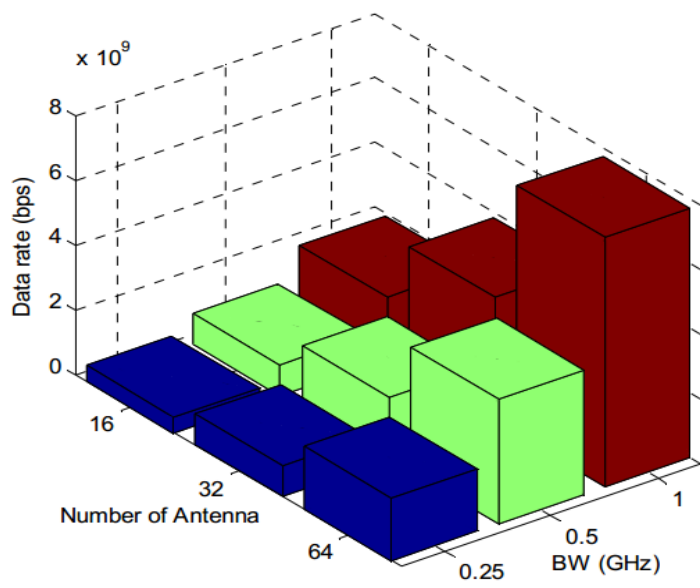

Figure 6. Data rate versus different BWs values for different number of antennas at $\mathrm{P}_{\mathrm{tx} \_\mathrm{BS} 1}=32.04 \mathrm{~dB}_{\mathrm{m}}$ and the lowest SINR (at edge of cell 1 )

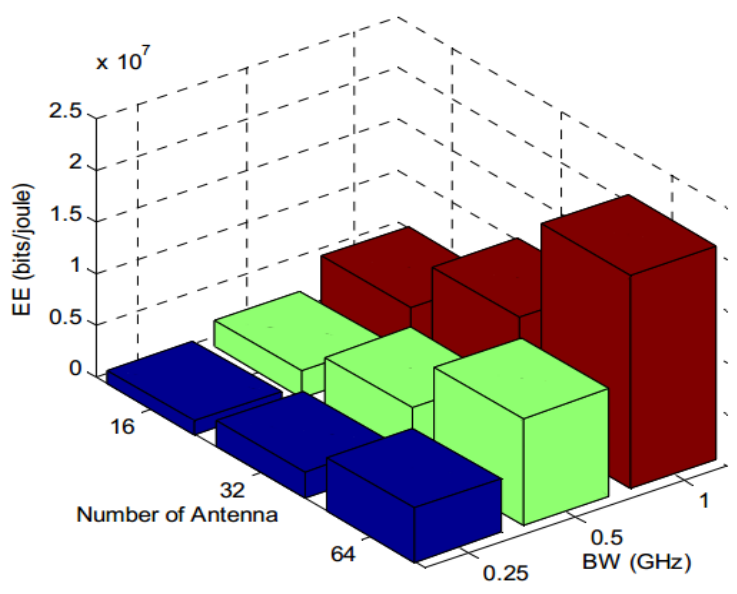

Figure 7. Energy efficiency versus different $\mathrm{BW}$ values for different number of antennas at $\mathrm{P}_{\mathrm{tx}_{\_} \mathrm{BS} 1}=\mathrm{P}_{\mathrm{tx} \_\mathrm{BS} 2}=\mathrm{P}_{\mathrm{tx} \_\mathrm{BS} 3}=32.04 \mathrm{~dB}_{\mathrm{m}}$ and at the lowest SINR (at edge of cell $1_{1}$ )

\section{CONCLUSION}

This paper presented a model and an investigation of the EE of multi-5G small cell system cellular networks. In addition, the concepts of new technologies of the large MIMO in the millimeter range communication, are considered. Since that the EE is a function of both the data rate and the total BS power consumption. Therefore, the EE improves with a larger BW size, a larger number of antennas, and decrease the total BS power consumption. The simulation results show that the large BW and the number of antennas combined with a high SINR increases the throughput, which increases the number of bits transferred per joule of energy and thus improves the EE of the cellular network.

\section{REFERENCES}

[1] Niklas Heuveldop, "Ericsson Mobility Report," Ericsson, 2017, [accessed 27 January 2018], [Online], Available: https://www.ericsson.com/assets/local/mobility-report/documents/2017/ericsson-mobility-report-june-2017.pdf.

[2] M.H. Alsharif and R. Nordin, "Evolution towards fifth generation (5G) wireless networks: Current trends and challenges in the deployment of millimetre wave, massive MIMO, and small cells," Telecommunication Systems, vol. 64, pp. 617-637, 2017.

[3] A. Abrol and R.K. Jha, "Power Optimization in 5G Networks: A Step Towards GrEEn Communication," in IEEE Access, vol. 4, pp. 1355-1374, 2016.

[4] A. Gupta and R.K. Jha, "A survey of 5g network: Architecture and emerging technologies," in IEEE Access, vol. 3, pp. 1206-1232, 2015.

[5] Pimmy Gandotra, Rakesh Jha, and Sanjeev Jain, "Green Communication in Next Generation Cellular Networks: A Survey," in IEEE Access, vol. 5, pp. 11727-11758, 2017.

[6] A.H. Jafari, D. López-Pérez, H. Song, H. Claussen, L. Ho, and J. Zhang, "Small cell backhaul: challenges and prospective solutions," EURASIP Journal on Wireless Communications and Networking, vol. 2015, no. 206, pp. 1-18, Aug. 2015.

[7] F. Han, S. Zhao, L. Zhang, and J. Wu, "Survey of strategies for switching off base stations in heterogeneous networks for greener 5G systems," in IEEE Access, vol. 4, pp. 4959-4973, 2016.

[8] A.I. Sulyman, A. Nassar, M.K. Samimi, G. Maccartney, T.S. Rappaport, and A. Alsanie, "Radio propagation path loss models for 5G cellular networks in the $28 \mathrm{GHZ}$ and $38 \mathrm{GHZ}$ millimeter-wave bands," in IEEE Communications Magazine, vol. 52, no. 9, pp. 78-86, Sep. 2014.

[9] I. Chih-Lin, C. Rowell, S. Han, Z. Xu, G. Li, and Z. Pan, "Toward green and soft: a 5G perspective," in IEEE Communications Magazine, vol. 52, no. 2, pp. 66-73, Feb. 2014.

[10] T. Chen, Y. Yang, H. Zhang, H. Kim, and K. Horneman, "Network energy saving technologies for green wireless access networks," in IEEE Wireless Communications, vol. 18, no. 5, pp. 30-38, Oct. 2011.

[11] H.Q. Ngo, E.G. Larsson, and T.L. Marzetta, "Energy and spectral efficiency of very large multiuser MIMO systems," in IEEE Transactions on Communications, vol. 61, no. 4, pp. 1436-1449, Apr. 2013.

[12] P.S. Parihar, R. Saraswat, and S. Maheshwari, "Energy and Spectral Efficiency of Very Large Multiuser MIMO Systems," International Journal of Computer Applications, vol. 111, no. 5, Feb. 2015.

[13] L. Zhao, K. Zheng, H. Long, H. Zhao, and W. Wang, "Performance analysis for downlink massive multiple-input multiple-output system with channel state information delay under maximum ratio transmission precoding," in IET Communications, vol. 8, no. 3, pp. 390-398, Feb. 2014. 
[14] X. Ge, C. Cao, M. Jo, M. Chen, J. Hu, and I. Humar, "Energy Efficiency Modelling and Analyzing Based on Multicell and Multi-antenna Cellular Networks," in KSII Transactions on Internet and Information Systems, vol. 4, no. 4, pp. 560-574, August 2010.

[15] A.A. Abdulkafi, T.S. Kiong, J. Koh, D. Chieng, A. Ting, and A.M. Ghaleb, "Energy efficiency of LTE macro base station," 2012 International Symposium on Telecommunication Technologies, Kuala Lumpur, pp. 259-264, 2012.

[16] G. Auer, V. Giannini, C. Desset, I. Godor, P. Skillermark, M. Olsson, et al., "How much energy is needed to run a wireless network?," in IEEE Wireless Communications, vol. 18, no. 5, pp. 40-49, Oct. 2011.

[17] W. Debus and L. Axonn, "RF Path Loss \& Transmission Distance Calculations," Axonn, LLC, Technical memorandukm, Aug. 2006.

[18] X. Ge, H. Cheng, M. Guizani, and T. Han, "5G wireless backhaul networks: challenges and research advances," in IEEE Network, vol. 28, no. 6, pp. 6-11, Nov.-Dec. 2014.

[19] C.R. Anderson and T.S. Rappaport, "In-building wideband partition loss measurements at 2.5 and $60 \mathrm{GHz}$," in IEEE Transactions on Wireless Communications, vol. 3, no. 3, pp. 922-928, May 2004.

[20] A.V. Alejos, M.G. Sánchez, and I. Cuiñas, "Measurement and analysis of propagation mechanisms at $40 \mathrm{GHz}$ : Viability of site shielding forced by obstacles," in IEEE Transactions on Vehicular Technology, vol. 57, no. 6, pp. 3369-3380, Nov. 2008.

[21] O. Edfors and F. Tufvesson, "Massive MIMO for next generation wireless systems," in IEEE Communications Magazine, vol. 52, no. 2, pp. 186-195, Feb. 2014.

[22] M.H. Alsharif, R. Nordin, N.F. Abdullah, and A.H. Kelechi, "How to make key 5G wireless technologies environmental friendly: A review," Transactions on Emerging Telecommunications Technologies, vol. 29, pp. e3254, 2018.

[23] M.H. Alsharif, R. Nordin, M.M. Shakir, and A.M. Ramly, "Small Cells Integration with the Macro-Cell Under LTE Cellular Networks and Potential Extension for 5G," Journal of Electrical Engineering \& Technology, vol. 14, pp. 2455-2465, 2019.

[24] M.H. Alsharif, A.H. Kelechi, J. Kim, and J.H. Kim, "Energy Efficiency and Coverage Trade-Off in 5G for EcoFriendly and Sustainable Cellular Networks," Symmetry, vol. 11, pp. 408, 2019.

[25] I. Khan, M.H. Alsharif, M.H. Zafar, M.O. Alassafi, M. Ashraf, Y. Huang, J. Kim, and J.H. Kim, "An Efficient Algorithm for mmWave MIMO Systems," Symmetry, vol. 11, pp. 786, 2019.

[26] S.L. Mohammed, M.H. Alsharif, S.K. Gharghan, I. Khan, and M. Albreem, "Robust Hybrid Beamforming Scheme for Millimeter-Wave Massive-MIMO 5G Wireless Networks," Symmetry, vol. 11, pp. 1424, 2019.

\section{BIOGRAPHIES OF AUTHORS}

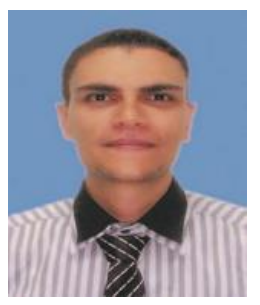

Mohammed H. Alsharif received his M.Sc and Ph.D in Electrical, Electronics and System Engineering from Universiti Kebangsaan Malaysia, Malaysia, in 2012 and 2015, respectively. Alsharif is an Assistant Professor in the Department of Electrical Engineering with Sejong University, South Korea since 2016. Currently, Alsharif is a visiting Professor with Department of Electrical and Electronics Engineering, Istanbul Gelisim University, Turkey. His research interest includes; wireless communication, 5G, IoT.

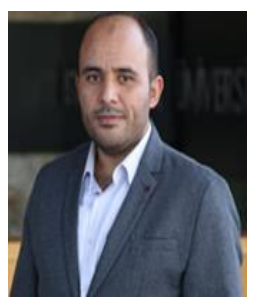

Khalid Yahya received his Ph.D. degree in Electrical Engineering from Kocaeli University, Kocaeli, Turkey, in 2018. He is presently working as an Assistant Professor of Mechatronics Engineering at Istanbul Gelisim University, Turkey. His current research interests include microelectronic circuit analysis and design, renewable energy resources, power electronics and MPPT designs for energy harvesting systems.

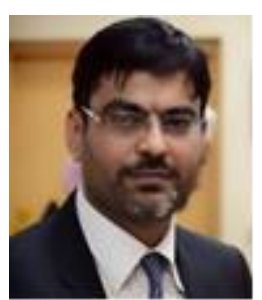

Shehzad Ashraf Chaudhry received the master's and Ph.D. degrees (with Distinction) from International Islamic University, Islamabad, Pakistan, in 2009 and 2016, respectively. He is currently with the Department of Computer Engineering, Faculty of Engineering and Architecture, Istanbul Gelisim University, Istanbul, Turkey. His current research interests include lightweight cryptography, elliptic/hyper elliptic curve cryptography, multimedia security 